\title{
Indirubin suppresses 4T1 murine breast cancer in vitro and in vivo by induction of ferroptosis and up- regulation of Ptgs2
}

\section{Xiuping Kuang}

Yunnan University of Traditional Chinese Medicine

\section{Yingnan Jiang}

Shenyang Pharmaceutical University

Jiwei Huang

Third Affiliated Hospital of Sun Yat-Sen University

\section{Yongzhi Guo}

Jinan University

weixi Li ( $D$ liweixi1001@163.com )

Yunnan University of Traditional Chinese Medicine https://orcid.org/0000-0002-6614-9508

\section{Research article}

Keywords: Breast cancer, indirubin, ferroptosis, Ptgs2, GSK-3 $\beta$

Posted Date: April 9th, 2021

DOl: https://doi.org/10.21203/rs.3.rs-390546/v1

License: (9) This work is licensed under a Creative Commons Attribution 4.0 International License. Read Full License 


\section{Abstract \\ Background}

Indirubin, isolated from Indigo Naturals, is reported to have the inhibitory activity of MCF-7 human breast cancer cells in vitro. However, studies on its anti-breast cancer activity in vivo and underlying mechanism are insufficient. We explored whether indirubin could trigger ferroptosis of breast cancer cells to exert antitumor activity.

\section{Methods}

Bioinformatical analysis was performed to detected the expression of prostaglandin-endoperoxide synthase 2 (Ptgs2) in breast cancer tissues Ptgs2-related prognosis for patients with breast cancer. Growth of 4T1 cells was assessed using wound healing assay and MTT assay. The levels of 4-HNE, GPX4, PTGS2 and GSK-3 $\beta$ proteins were detected by Western blot, and the mRNA of Ptgs 2 was tested by qPCR. The GSH and MDA were determined by commercial kits. Molecular docking was employed to study interaction between indirubin and GSK-3 $\beta$. An 4T1 murine breast cancer was adopted to evaluate the in vivo antitumor activity of indirubin.

\section{Results}

Indirubin promoted ferroptosis of $4 \mathrm{~T} 1$ breast cancer cells with deplete of $\mathrm{GSH}$, increased MDA and 4-HNE level, as well as decreased GPX4 expression. Indirubin suppressed the growth of 4T1 breast tumor in vivo. Mechanism study showed indirubin up regulated Ptgs2 expression by promoting phosphorylation (Ser 9) of GSK-3ß.

\section{Conclusions}

Indirubin suppresses 4T1 murine breast cancer in vitro and in vivo by induction of ferroptosis and upregulation of Ptgs2.

\section{Background}

Breast cancer is the most common cancer among women with increasing incidence year by year. Furthermore, it is also one of the leading causes of death among women with cancer worldwide [1-3]. Chemotherapy is one of the important methods for the treatment of breast cancer, but the drug resistance and side effects make it imperative to find new breast cancer treatment drugs [4-6].

Indigo Naturalis, the classical Chinese medicine, has been used in China for many centuries, and modern pharmacological studies have shown that it has anti-inflammatory, antiviral, antibacterial, anti-tumor and 
anti-psoriatic activities [7-11]. In the study of the pharmacological active components of Indigo Naturalis, indirubin has received the most attention [12]. Indirubin, the major active component of Indigo Naturalis, have been widely known for its clinical use for treatment of chronic myelocytic leukemia (CML) $[13,14]$. Recent studies have shown that indirubin can also be used to treat other types of cancer, like breast cancer [15-17]. However, the research on the mechanism of indirubin in the treatment of breast cancer is still insufficient.

Ferroptosis, a new type of cell death, is mediated by iron-dependent accumulation of lipid peroxidation. It was found that ferroptosis can be involved in the occurrence, progression and treatment of tumor. And emerging evidence indicates triggering ferroptosis of cancer cells is an important means for cancer therapy [18-21].

In this study, we aimed to explore whether indirubin could play an anti-breast cancer role by inducing the occurrence of ferroptosis. We analyzed the clinical data provided by NCBI database and found that breast cancer was related to the low level of prostaglandin-endoperoxide synthase 2 (Ptgs2), a molecular marker of ferroptosis. In vivo and in vitro studies suggested that indirubin could exert the anti-breast cancer effect by up-regulating PTGS2 and inducing ferroptosis.

\section{Methods}

\section{Chemicals and reagents}

Indirubin (wkq-01742, purity $\geq 97.0 \%, \mathrm{HPLC}$ ) and indigo (wkq-00174, purity $\geq 98.0 \%$, HPLC) were purchased from Sichuan Weikeqi Biological Technology Co., Ltd (Chengdu, China). Primary antibodies to PTGS2 (GB11077-2) was purchased from Servicebio Co., Ltd (Wuhan, China). Primary antibodies, anti-

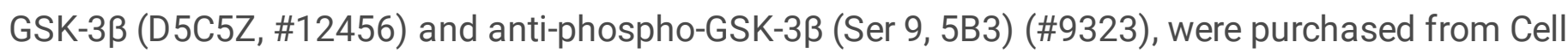
Signaling Technology (CST) (Boston, MA, USA). Primary antibodies to 4-HNE (ab46545) and GPX4 (ab125066) were purchased from Abcam (Cambridge, MA, USA). Adriamycin (A183027, purity $\geq 97.0 \%$ ) was purchased from Shanghai Aladdin Bio-Chem Technology Co., LTD (Shanghai, China). Antiglyceraldehyde 3-phosphate dehydrogenase (GAPDH) antibody (FD0063) was from Fude Biological Technology (Hangzhou, China). Other chemicals were purchased from standard commercial suppliers.

\section{Bioinformatical analysis of breast cancer-related genes}

The gene expression profile of GSE20713, GSE42568 and GSE 54002 in breast cancer and normal breast tissues were obtained from the free public database, NCBI-GEO database. Differentially expressed genes (DEGs) between breast cancer specimen and normal breast specimen were identified via GEO2R online tools with $\log \mathrm{FC}<-2$ and adjust $P$ value $<0.05$ [22]. Then, the raw data in TXT format were checked in Venn software online to detect the commonly DEGs among the three datasets. The prognostic relationships between selected genes and breast cancer were analyzed by Kaplan-Meier plotter and GEPIA online tools $[23,24]$.

\section{Cell culture}


4T1 mouse breast cancer cell was purchased from the Cell Bank of the China Academy of Sciences and cultured in DMEM medium (C11995500BT, Gibco, Carlsbad, CA, USA) supplemented with 10\% fetal bovine serum (FBS) (ST40-39500, PAN-Seratech, Aidenbach, Germany) and 1\% penicillin-streptomycin (C0222, Beyotime, Shanghai, China) at $37^{\circ} \mathrm{C}$ under a humidified $5 \% \mathrm{CO}_{2}$ atmosphere.

\section{Cell viability analysis}

4T1 cells were seeded in a 96-well plate and cultured in DMEM supplemented with 10\% FBS overnight. After $24 \mathrm{~h}$ of drug treatment, 3-(4,5-dimethyl-2-thiazolyl)-2,5-diphenyl-2-H-tetrazolium bromide (MTT) solution was added into each well and incubated at $37^{\circ} \mathrm{C}$ for $4 \mathrm{~h}$. The medium was removed and formazan crystals were later dissolved in dimethyl sulfoxide (DMSO). The absorbance was measured at $570 \mathrm{~nm}$.

\section{Wound healing assay}

4T1 cells were seeded into 12-well plates and grown to a monolayer until $90 \%$ confluence. After removing the medium, the cell monolayer was scraped in a straight line with a sterile tip of 200 microliters. Next, the isolated cells were washed with phosphate buffer saline (PBS). The cells were treated with fresh medium containing $2 \%$ FBS with different drug concentrations for $48 \mathrm{~h}$. Untreated cells served as the control. All the cells were photographed with a microscope before and after the drug treatment. Image $\mathrm{J}$ software was used to quantitatively calculate the wound area.

\section{Animal experiment}

BALB/c mice (6-8 weeks old, female) were purchased from Guangdong Medical Laboratory Animal Center (Guangzhou, China) with permission No. SCXK 2017 - 0174. Animal experiments were approved by the Animal Care and Use Committee of Jinan University and conducted in accordance with National Institute of Health's Guide for the Care and Use of Laboratory Animals (7th edition, United States). All mice were kept in a non-pathogenic animal chamber with a temperature of $23 \pm 1^{\circ} \mathrm{C}$ and a dark period of $12 \mathrm{~h}$, and fed with standard laboratory diet and water. The animals were allowed to acclimatize for a week before the experiment. Mice received a subcutaneous injection with 4 T 1 cells $\left(2 \times 10^{6}\right.$ cells) into the right flank. When the tumor diameter reached about $5 \mathrm{~mm}$, the mice were randomly divided into 5 groups. The mice were treated with saline, indigo (20 mg/kg), indirubin (low dose, $10 \mathrm{mg} / \mathrm{kg}$; high dose, $20 \mathrm{mg} / \mathrm{kg}$ ) and Adriamycin ( $1 \mathrm{mg} / \mathrm{kg}$, clinically used in the treatment of breast cancer, as a positive control drug) every day (Supplementary Table 1). The tumor sizes were measured every 2 day using calipers, and the tumor volume was calculated by the following formula: volume $\left(\mathrm{mm}^{3}\right)=0.5 \times[$ length $(\mathrm{mm})] \times[\text { width }(\mathrm{mm})]^{2}$. The tumor size at 21 th day post-injection was used as the endpoint reading. Mice were sacrificed with diethyl ether anesthesia. The data were presented as the mean volume $\mathrm{X} \pm \mathrm{S}$. $\mathrm{E}$.

\section{Histological analysis}

Breast tumors were fixed with $4 \%$ paraformaldehyde for $48 \mathrm{~h}$ at room temperature. Then, they were dehydrated, transparent, paraffin-embedded and cut into $5 \mu \mathrm{m}$ thick slices. Hematoxylin and eosin (H\&E) 
were used for staining of tumor tissues. The histological changes were observed and imaged at 40x magnification under an automatic scanning microscope (PreciPoint M8, Freising, Germany).

\section{Western blotting}

The cells and tumor tissues were lysed using RIPA (P0013C, Beyotime) lysis buffer containing $1 \mathrm{mM}$ PMSF (P1005, Beyotime) on ice for $30 \mathrm{~min}$. After centrifugation at $12,000 \times g$ at $4{ }^{\circ} \mathrm{C}$ for $10 \mathrm{~min}$, the supernatants were collected and total protein concentrations were determined using BCA protein assay kit (Pierce, Rockford, IL, USA). The protein samples were separated by $10 \%$ SDS-PAGE and then transferred to PVDF membranes (Millipore Corporation, Billerica, MA, USA). The membranes were blocked with $5 \%$ skim milk dissolved in TBST buffer at room temperature for $1 \mathrm{~h}$ and probed with the indicated primary antibodies at $4{ }^{\circ} \mathrm{C}$ overnight and then incubated with HRP-labeled secondary antibodies at room temperature for $2 \mathrm{~h}$. Target proteins were detected using ECL Western Blotting Detection Reagent (20190120, Fude Biological Technology) and visualized by Tanon 5200 imaging system (Tanon, Shanghai, China). GAPDH is used as an internal control.

\section{Reverse transcription and quantitative real-time PCR}

Total RNA of cells was extracted using TRIzol reagent (Invitrogen, Carlsbad, CA, USA) according to manufacturer's instructions. RNA concentrations were determined by optical density measurement at $260 \mathrm{~nm}$ on a spectrophotometer (NanoDrop 2000, Thermo Scientific; Wilmington, DE, USA). cDNA was synthesized from the purified RNA by both random and oligo (dT) priming by a TransScript One-Step gDNA Removal and cDNA Synthesis SuperMix kit (AT311, TransGen Biotech, Beijing, China) and amplified in real-time quantitative PCR by a TransStart Top Green qPCR SuperMix kit (AQ131, TransGen Biotech). The following primer sequences were used: Ptgs2, 5'-CCACTTCAAGGGAGTCTGGA-3' (Forward), Ptgs2, 5'-AGTCATCTGCTACGGGAGGA-3' (Reverse); Gapdh, 5'-AACTTTGGCATTGTGGAAGG-3' (Forward), Gapdh, 5'-GGATGCAGGGATGATGTTCT-3' (Reverse). Using the comparative threshold cycle (Ct), relative expression was calculated using $2^{-\triangle \Delta C t}$ method and normalized by the expressions of Gapdh from the same samples.

\section{Determination of glutathione (GSH) and malondialdehyde (MDA)}

The cells and tumor tissues were lysed using RIPA lysis buffer containing $1 \mathrm{mM}$ PMSF on ice for $30 \mathrm{~min}$ and then centrifuged at $12,000 \times g$ at $4{ }^{\circ} \mathrm{C}$ for $10 \mathrm{~min}$. Then, the resulting cell lysates were utilized to assess GSH content and MDA content, using commercially test kits (GSH, A061-1-1, Nanjing Jiancheng BioTechnology, Nanjing, China; MDA, S0131, Beyotime), respectively, according to the manufacturer's protocols.

\section{Molecular docking}

The molecular docking method was used for the analysis of indirubin and indigo binding with GSK-3ß. The discovery Studio 3.0 docking program was adopted [25]. The structure of GSK-3 $\beta$ was downloaded 
from PDB database (PDB ID: 205K). The preparation of protein structure included adding hydrogen atoms, removing water molecules, and assigning Charmm forcefield. All parameters were set as default.

\section{Statistical analysis}

Statistical analysis was performed using GraphPad Prism 6.0 software (San Diego, CA, USA). The statistical significance of differences between two groups was determined with unpaired Student's $t$-test and multiple comparisons were analyzed with one-way ANOVA. All data presented as mean \pm standard deviation. Differences were considered statistically significant at $p<0.05$.

\section{Results}

\section{Low expression of Ptgs2 in breast cancer}

There were 587, 343 and 564 DEGs between breast tumors and normal breast tissues from GSE20713, GSE154002 and GSE42568, respectively, via GEO2R online tools. Then, we used Venn diagram software to identify the commonly down-regulated genes in the three datasets. Results showed that a total of 16 down-regulated genes $(\log F C<-2, P<0.05)$ was identified in the breast cancer tissues (Fig. 1A, B). Noticeably, Ptgs2, a ferroptosis marker, was down-regulated in breast cancer patients, which indicated that ferroptosis may be related to breast cancer [26, 27]. Furthermore, the prognostic information of Ptgs2 was analyzed employing the Kaplan-Meier plotter. Result showed that the low mRNA expression of Ptgs2 (HR, 0.82; $\mathrm{Cl}, 0.7-0.96)$ was associated with the poorer overall survival for patients with breast cancer (Fig. 1C). In addition, we also performed GEPIA and found that Ptgs2 mRNA was significantly lower in breast cancer tissues than that in normal tissues $(p<0.05)$ (Fig. 1D). Results above showed the low expression of Ptgs 2 in breast cancer.

\section{Indirubin induces ferroptosis of breast cancer cell in vitro}

In order to investigate whether indirubin has the inhibitory effect on breast cancer, we firstly performed wound healing assay to study the effect of indirubin on growth of $4 \mathrm{~T} 1$ cells in vitro. As shown in Fig. $2 \mathrm{~A}$ and $\mathrm{B}$, indirubin $(40 \mu \mathrm{M}$ and $80 \mu \mathrm{M})$ significantly inhibited wound closure of $4 \mathrm{~T} 1$ cells $(p<0.05, p<0.01)$. Next, we detected the effect of indirubin on viability of $4 \mathrm{~T} 1$ cells by MTT assay. Result showed that the viability of $4 \mathrm{~T} 1$ cells was significantly inhibited in a dose-dependent manner after incubation with indirubin (Fig. 2C). These results demonstrated the anti-breast cancer effect of indirubin in vitro. Then, we explored whether ferroptosis was involved in the anti-breast cancer effect of indirubin. The pre-treatment of Fer-1 (ferrostatin-1, ferroptosis-specific inhibitor, $25 \mu \mathrm{M}$ ) significantly reversed indirubin-induced inhibition of $4 \mathrm{~T} 1$ cells viability $(p<0.05)$ (Fig. 2D). However, Nec-1 (necrosis inhibitor, $50 \mu \mathrm{M}$ ) and z-VAD (apoptosis inhibitor, $50 \mu \mathrm{M}$ ) didn't protect 4T1 cells from indirubin-induced inhibition of viability (Fig. 2D). These results indicated indirubin induced ferroptosis of breast cancer cell in vitro. The occurrence of ferroptosis is characterized by the depletion of GSH and accumulation of lipid peroxides. Meanwhile, the result showed that indirubin $(40 \mu \mathrm{M}$ and $80 \mu \mathrm{M})$ reduced the GSH content of $4 \mathrm{~T} 1$ cells $(p<0.05, p<0.05)$ (Fig. 2E). MDA and 4-hydroxynonenal (4-HNE) are major end products derived from lipid peroxides. As 
shown in Fig. $2 \mathrm{~F}$ and $\mathrm{G}$, indirubin $(80 \mu \mathrm{M})$ significantly increased the level of MDA $(p<0.05)$ and $4-\mathrm{HNE}(p$ $<0.05$ ). In addition, glutathione peroxidase 4 (GPX4) plays a key role in reducing lipid peroxides. The expression level of GPX4 significantly decreased by indirubin $(40 \mu \mathrm{M}$ and $80 \mu \mathrm{M})(p<0.05, p<0.05)$ (Fig. 2G). These results indicated that indirubin induced ferroptosis of breast cancer cell in vitro.

\section{Indirubin up-regulates the expression of PTGS2 in breast cancer cell}

Given that low expression of Ptgs2 in breast cancer found by bioinformatics analysis in Fig. 1, We sought to explore whether indirubin-induced ferroptosis was related to PTGS2. We firstly detected the effects of indirubin on the expression of PTGS2 by qPCR and Western blotting. As shown in Fig. 3A, indirubin (40 $\mu \mathrm{M}$ and $80 \mu \mathrm{M})$ significantly elevated the mRNA level of Ptgs2 in 4T1 cells $(p<0.01, p<0.001)$. Moreover, indirubin $(80 \mu \mathrm{M})$ significantly increased the expression level of PTGS2 protein $(p<0.05)$ (Fig. 3B).

Indirubin was reported as the potent inhibitor of glycogen synthase kinase-3 beta (GSK-3 $\beta$ ) [28]. We found that indirubin could form hydrogen bonds with Arg141, Gln185 and Val135 of GSK-3 $\beta$ protein by molecular docking (Fig. 3C, D). Interestingly, indigo, a main component from Indigo Naturalis and isomer of indirubin, only forms the hydrogen bond with Arg141 of GSK-3ß (Fig. 3C, D). It was indicated that two carbonyl groups in opposite positions of indirubin benefited its binding with GSK-3 $\beta$. We further analyzed the effect of indirubin on phosphorylation (Ser 9) of GSK-3 $\beta$ in 4T1 cells by western blotting. Indirubin but indigo could promote phosphorylation (Ser 9) of GSK-3 $\beta$ (Fig. 3E). Correspondingly, indigo had no effect on the mRNA and protein expression of PTGS2 (Fig. 3F, G). In addition, indigo failed to triggering lipid peroxidation and ferroptosis (Fig. $3 \mathrm{H}$ and Supplementary Fig. 1). Indeed, it has been reported that GSK-3 $\beta$ participates in regulating the expression of PTGS2 $[29,30]$. Hence, we speculated that indirubin may regulate the activity of GSK-3 $\beta$ to promote the expression of PTGS2 and then trigger the ferroptosis of tumor cells.

\section{Indirubin suppresses breast cancer in vivo}

Indirubin has been shown in vitro to induce ferroptosis of breast cancer cells, which may concern upregulation of Ptgs2. Therefore, we next sought to investigate whether indirubin could suppress breast cancer in vivo. Firstly, we evaluated the anti-tumor activity of indirubin using the mouse model of breast cancer by injection of $4 \mathrm{~T} 1$ cells subcutaneously. Results showed that indirubin $(20 \mathrm{mg} / \mathrm{kg})$ significantly inhibited the growth of breast cancer with reduced tumor weight $(p<0.05)$ and volume $(p<0.01)$ compared with the control group (Fig. 4A-C). Histopathological observation of tumor tissue showed that indirubin resulted in breast tumor necrosis (Fig. 4D). Meanwhile, the positive drug ADR suppressed breast cancer (Fig. 4A-D). After indirubin $(20 \mathrm{mg} / \mathrm{kg})$ treatment, the $\mathrm{GSH}$ content of tumor tissues significantly decreased $(p<0.05)$ (Fig. 4F). Indirubin $(10 \mathrm{mg} / \mathrm{kg}$ and $20 \mathrm{mg} / \mathrm{kg})$ significantly increased the MDA level of tumor tissues $(p<0.01, p<0.01$ ) (Fig. 4G). In addition, indirubin also increased the 4-HNE level of tumor tissues, and decreased the expression of GPX4 of tumor tissues (Fig. 4E). As expected, Indirubin $(10 \mathrm{mg} / \mathrm{kg}$ and $20 \mathrm{mg} / \mathrm{kg})$ significantly increased the level of PTGS2 protein of tumor tissues $(p<0.01, p$ 
$<0.001)$ (Fig. 4H). However, treatment of indigo $(20 \mathrm{mg} / \mathrm{kg})$ didn't decrease the weight and volume of breast tumors and promote necrosis and lipid peroxidation of tumors consistent with in vitro results (Fig. 4). The results showed that indirubin promoted lipid peroxidation of tumors suppressed breast cancer in vivo.

\section{Discussion}

Breast cancer is one of the leading causes of cancer death in women. Chemotherapy is one of the effective but risky ways to treat breast cancer. Naturally derived compounds have the potential to develop new drugs because of their safety and efficacy. Indirubin and derivatives has the inhibitory effect on tumors [15-17]. In our study, effectiveness and pharmacological mechanism of indirubin was explored, which may contribute to development of anti-breast cancer drug.

Ferroptosis is a unique way of cell death, and it has been shown that ferroptosis plays an important role in the occurrence and development of tumors [31]. By inducing ferroptosis of tumor cells, it can be used for tumor therapy [32,33]. Ptgs2, also known as cyclooxygenase-2 (Cox-2), is the key enzyme in prostaglandin biosynthesis. As a matter of fact, cyclooxygenases can catalyze lipid oxidation [34, 35]. Studies have shown that Ptgs2 is involved in the process of ferroptosis, and Ptgs2 is significantly upregulated in the ferroptosis induced by RSL3 and erastin [36]. Meanwhile, suppression of Ptgs2 can inhibit the ferroptosis of cells and play a protective role $[26,27]$. In our study, results of bioinformatical analysis showed that ferroptosis-related gene Ptgs 2 was expressed lower in breast cancer, which indicated an efficient strategy for treating breast cancer. It is possible to induce ferroptosis of breast cancer cells by ug-regulation of Ptgs2. Then, in vivo and in vitro analysis results showed that indirubin could play an anti-breast cancer role by upregulation of Ptgs 2 to induce ferroptosis.

GSK-3 $\beta$ is a protein related to the regulation of Ptgs2, which can increase the expression of Ptgs 2 by inhibiting GSK-3 $\beta$ activity [30]. The activity of GSK-3 $\beta$ is regulated by phosphorylation at Ser 9 and Tyr 216 in opposing directions, with phosphorylation of Ser 9 decreasing GSK-3 $\beta$ activity and phosphorylation of Tyr 216 increasing GSK-3 $\beta$ activity [37]. However, previous studies have shown that indirubin is an inhibitor of GSK-3 $\beta$ [28]. Our results showed that indirubin promoted phosphorylation of GSK-3 $\beta$ at Ser 9 and then inhibited the activity of GSK-3ß. Molecular docking further proved that indirubin with two carbonyl groups at different sides could form additional hydrogen bonds with GIn185 and Val135 of GSK-3 $\beta$, compared to indigo with two carbonyl groups the same side indicating the special role of indirubin in regulating GSK-3 $\beta$.

\section{Conclusion}

In conclusion, indirubin suppresses breast cancer by up-regulation of Ptgs2 and induction of ferroptosis in vitro and in vivo in a 4T1 murine breast cancer model, which may be related bind of indirubin with GSK$3 \beta$ and then promote its phosphorylation at Ser 9 (Fig. 5). 


\section{Declarations}

\section{Acknowledgements}

Not applicable.

\section{Authors' Contributions}

X.P. Kuang performed the experiments, acquired and analyzed all the data. Y.N. Jiang and J.W. Huang drafted the manuscript. W.X. Li and Y.Z. Guo designed the research, revised and approved the manuscript. All authors read and approved final manuscript.

\section{Funding}

This work was supported by the Yunnan Provincial Science and Technology Department-Applied Basic Research Joint Special Funds of Yunnan University of Chinese Medicine (2019FF002 (-007), 2017FF116 (-015)); National Natural Science Foundation of China $(81560661,81960780)$. They had no role in the design of the study; collection, analysis, and interpretation of the data; or writing of the manuscript.

\section{Availability of data and materials}

The datasets used and/or analyzed during the current study are available from the corresponding author on reasonable request.

\section{Ethics approval and consent to participate}

Animal experiments were approved by the Animal Care and Use Committee of Jinan University and conducted in accordance with National Institute of Health's Guide for the Care and Use of Laboratory Animals (7th edition, United States).

\section{Consent for publication}

Not applicable.

\section{Competing interests}

The authors declare that they have no competing interests. 


\section{Abbreviations}

4-HNE, 4-hydroxynonenal; Cox-2, cyclooxygenase-2; CML, chronic myelocytic leukemia; DEGs, differentially expressed genes; DMSO, dimethyl sulfoxide; FBS, fetal bovine serum; GPX4, glutathione peroxidase 4; GSH, glutathione; GSK-3 $\beta$, Glycogen synthase kinase $3 \beta ; \mathrm{H} \& \mathrm{E}$, hematoxylin and eosin; MDA, malondialdehyde; MTT, 3-(4,5-dimethyl-2-thiazolyl)-2,5-diphenyl-2-H-tetrazolium bromide; PBS, phosphate buffer saline; Ptgs2, prostaglandin-endoperoxide synthase 2.

\section{References}

1. Porter PL: Global trends in breast cancer incidence and mortality. Salud Publica Mex 2009, 51:s141s146. https://doi.org/10.1590/s0036-36342009000800003

2. DeSantis CE, Bray F, Ferlay J, Lortet-Tieulent J, Anderson BO, Jemal A: International variation in female breast cancer incidence and mortality rates. Cancer Epidemiol Biomarkers Prev 2015, 24(10):1495-1506. https://doi.org/10.1158/1055-9965.epi-15-0535

3. Ghoncheh M, Pournamdar Z, Salehiniya H: Incidence and mortality and epidemiology of breast cancer in the world. Asian Pac J Cancer Prev 2016, 17(S3):43-46. https://doi.org/10.7314/apjcp.2016.17.s3.43

4. Marquette C, Nabell L: Chemotherapy-resistant metastatic breast cancer. Curr Treat Options Oncol 2012, 13(2):263-275. https://doi.org/10.1007/s11864-012-0184-6

5. Naito Y, Kai Y, Ishikawa T, Fujita T, Uehara K, Doihara H, Tokunaga S, Shimokawa M, Ito Y, Saeki T: Chemotherapy-induced nausea and vomiting in patients with breast cancer: a prospective cohort study. Breast Cancer 2020, 27(1):122-128. https://doi.org/10.1007/s12282-019-01001-1

6. Partridge AH, Burstein HJ, Winer EP: Side effects of chemotherapy and combined chemohormonal therapy in women with early-stage breast cancer. J Natl Cancer Inst Monogr 2001, 2001(30):135-142. https://doi.org/10.1093/oxfordjournals.jncimonographs.a003451

7. Kawai S, lijima H, Shinzaki S, Hiyama S, Yamaguchi T, Araki M, Iwatani S, Shiraishi E, Mukai A, Inoue $\mathrm{T}$ : Indigo Naturalis ameliorates murine dextran sodium sulfate-induced colitis via aryl hydrocarbon receptor activation. J Gastroentero/2017, 52(8):904-919. https://doi.org/10.1007/s00535-016-1292z

8. Zhang T, Huang H-z, Xu R-c, Wang J-b, Yang M, Cao J-h, Zhang Y, Zhang D-k, Han L: An anti-influenza virus activity-calibrated chemical standardization approach for quality evaluation of indigo naturalis. Anal Methods 2019, 11(37):4719-4726.

9. Chiang Y-R, Li A, Leu Y-L, Fang J-Y, Lin Y-K: An in vitro study of the antimicrobial effects of indigo naturalis prepared from Strobilanthes formosanus Moore. Molecules 2013, 18(11):14381-14396. https://doi.org/10.3390/molecules181114381

10. Wang L, Zhou G-B, Liu P, Song J-H, Liang Y, Yan X-J, Xu F, Wang B-S, Mao J-H, Shen Z-X: Dissection of mechanisms of Chinese medicinal formula Realgar-Indigo naturalis as an effective treatment for 
promyelocytic leukemia. Proc Natl Acad Sci USA 2008, 105(12):4826-4831.

https://doi.org/10.1073/pnas.0712365105

11. Cheng H-M, Wu Y-C, Wang Q, Song M, Wu J, Chen D, Li K, Wadman E, Kao S-T, Li T-C: Clinical efficacy and IL-17 targeting mechanism of Indigo naturalis as a topical agent in moderate psoriasis. BMC Complement Altern Med 2017, 17(1):1-11. https://doi.org/10.1186/s12906-017-1947-1

12. Gaboriaud-Kolar N, Vougogiannopoulou K, Skaltsounis A-L: Indirubin derivatives: A patent review (2010-present). Expert Opin Ther Pat 2015, 25(5):583-593. https://doi.org/10.1517/13543776.2015.1019865

13. Hoessel R, Leclerc S, Endicott JA, Nobel ME, Lawrie A, Tunnah P, Leost M, Damiens E, Marie D, Marko D: Indirubin, the active constituent of a Chinese antileukaemia medicine, inhibits cyclin-dependent kinases. Nat Cell Bio/ 1999, 1(1):60-67. https://doi.org/10.1038/9035

14. Xiao Z, Hao Y, Liu B, Qian L: Indirubin and meisoindigo in the treatment of chronic myelogenous leukemia in China. Leuk Lymphoma 2002, 43(9):1763-1768.

https://doi.org/10.1080/1042819021000006295

15. Aobchey P, Sinchaikul S, Phutrakul S, Chen S-T: Simple purification of indirubin from Indigofera tinctoria Linn. and inhibitory effect on MCF-7 human breast cancer cells. Chiang Mai J Sci 2007, 34:329-337.

16. Braig S, Kressirer CA, Liebl J, Bischoff F, Zahler S, Meijer L, Vollmar AM: Indirubin derivative 6BIO suppresses metastasis. Cancer Res 2013, 73(19):6004-6012. https://doi.org/10.1158/00085472.can-12-4358

17. Nam S, Buettner R, Turkson J, Kim D, Cheng JQ, Muehlbeyer S, Hippe F, Vatter S, Merz K-H, Eisenbrand G: Indirubin derivatives inhibit Stat3 signaling and induce apoptosis in human cancer cells. Proc Natl Acad Sci USA 2005, 102(17):5998-6003. https://doi.org/10.1073/pnas.0409467102

18. Eling N, Reuter L, Hazin J, Hamacher-Brady A, Brady NR: Identification of artesunate as a specific activator of ferroptosis in pancreatic cancer cells. Oncoscience 2015, 2(5):517. https://doi.org/10.18632/oncoscience.160

19. Louandre C, Ezzoukhry Z, Godin C, Barbare JC, Mazière JC, Chauffert B, Galmiche A: Iron-dependent cell death of hepatocellular carcinoma cells exposed to sorafenib. Int J Cancer2013, 133(7):17321742. https://doi.org/10.1002/ijc.28159

20. Ma S, Dielschneider RF, Henson ES, Xiao W, Choquette TR, Blankstein AR, Chen Y, Gibson SB: Ferroptosis and autophagy induced cell death occur independently after siramesine and lapatinib treatment in breast cancer cells. PLoS One 2017, 12(8):e0182921. https://doi.org/10.1371/journal.pone.0182921

21. Chen G-Q, Benthani FA, Wu J, Liang D, Bian Z-X, Jiang X: Artemisinin compounds sensitize cancer cells to ferroptosis by regulating iron homeostasis. Cell Death Differ 2020, 27(1):242-254. https://doi.org/10.1038/s41418-019-0352-3

22. Davis S, Meltzer PS: GEOquery: a bridge between the Gene Expression Omnibus (GEO) and BioConductor. Bioinformatics 2007, 23(14):1846-1847. 
https://doi.org/10.1093/bioinformatics/btm254

23. Hou G-X, Liu P, Yang J, Wen S: Mining expression and prognosis of topoisomerase isoforms in nonsmall-cell lung cancer by using Oncomine and Kaplan-Meier plotter. PloS One 2017, 12(3):e0174515. https://doi.org/10.1371/journal.pone.0174515

24. Tang Z, Li C, Kang B, Gao G, Li C, Zhang Z: GEPIA: a web server for cancer and normal gene expression profiling and interactive analyses. Nucleic Acids Res 2017, 45(W1):W98-W102. https://doi.org/10.1093/nar/gkx247

25. Wu G, Robertson DH, Brooks III CL, Vieth M: Detailed analysis of grid-based molecular docking: A case study of CDOCKER-A CHARMm-based MD docking algorithm. J Comput Chem 2003, 24(13):1549-1562. https://doi.org/10.1002/jcc.10306

26. Li Q, Han X, Lan X, Gao Y, Wan J, Durham F, Cheng T, Yang J, Wang Z, Jiang C: Inhibition of neuronal ferroptosis protects hemorrhagic brain. JCl Insight 2017, 2(7). https://doi.org/10.1172/jci.insight.90777

27. Xiao X, Jiang Y, Liang W, Wang Y, Cao S, Yan H, Gao L, Zhang L: miR-212-5p attenuates ferroptotic neuronal death after traumatic brain injury by targeting Ptgs2. Mol Brain 2019, 12(1):78. https://doi.org/10.1186/s13041-019-0501-0

28. Leclerc S, Garnier M, Hoessel R, Marko D, Bibb JA, Snyder GL, Greengard P, Biernat J, Wu Y-Z, Mandelkow E-M: Indirubins Inhibit Glycogen Synthase Kinase-3 $\beta$ and CDK5/P25, Two Protein Kinases Involved in Abnormal Tau Phosphorylation in Alzheimer's Disease A PROPERTY COMMON TO MOST CYCLIN-DEPENDENT KINASE INHIBITORS? J Biol Chem 2001, 276(1):251-260. https://doi.org/10.1074/jbc.m002466200

29. Ye Z, Wang N, Xia P, Wang E, Yuan Y, Guo Q: Delayed administration of parecoxib, a specific COX-2 inhibitor, attenuated postischemic neuronal apoptosis by phosphorylation Akt and GSK-3ß. Neurochem Res 2012, 37(2):321-329. https://doi.org/10.1007/s11064-011-0615-y

30. Bai L, Chang H-M, Cheng J-C, Chu G, Leung PC, Yang G: Lithium Chloride Increases COX-2 Expression and PGE2 Production in a Human Granulosa-Lutein SVOG Cell Line Via a GSK-3 $\beta / \beta$-Catenin Signaling Pathway. Endocrinology 2017, 158(9):2813-2825. https://doi.org/10.1210/en.2017-00287

31. Lu B, Chen XB, Ying MD, He QJ, Cao J, Yang B: The role of ferroptosis in cancer development and treatment response. Front Pharmaco/2018, 8:992. https://doi.org/10.3389/fphar.2017.00992

32. Shen Z, Song J, Yung BC, Zhou Z, Wu A, Chen X: Emerging strategies of cancer therapy based on ferroptosis. Adv Mater 2018, 30(12):1704007. https://doi.org/10.1002/adma.201704007

33. Lachaier E, Louandre C, Godin C, Saidak Z, Baert M, Diouf M, Chauffert B, Galmiche A: Sorafenib induces ferroptosis in human cancer cell lines originating from different solid tumors. Anticancer Res 2014, 34(11):6417-6422.

34. Liu X, Moon SH, Jenkins CM, Sims HF, Gross RW: Cyclooxygenase-2 mediated oxidation of 2arachidonoyl-lysophospholipids identifies unknown lipid signaling pathways. Cell Chem Biol 2016, 23(10):1217-1227. https://doi.org/10.1016/j.chembiol.2016.08.009 
35. Bretscher P, Egger J, Shamshiev A, Trötzmüller M, Köfeler H, Carreira EM, Kopf M, Freigang S: Phospholipid oxidation generates potent anti-inflammatory lipid mediators that mimic structurally related pro-resolving eicosanoids by activating Nrf2. EMBO Mol Med 2015, 7(5):593-607. https://doi.org/10.15252/emmm.201404702

36. Yang WS, SriRamaratnam R, Welsch ME, Shimada K, Skouta R, Viswanathan VS, Cheah JH, Clemons PA, Shamji AF, Clish CB: Regulation of ferroptotic cancer cell death by GPX4. Cel/ 2014, 156(1-2):317331. https://doi.org/10.1016/j.cell.2013.12.010

37. Forde Ja, Dale TC: Glycogen synthase kinase 3: a key regulator of cellular fate. Cell Mol Life Sci 2007, 64(15):1930-1944. https://doi.org/10.1007/s00018-007-7045-7

\section{Figures}
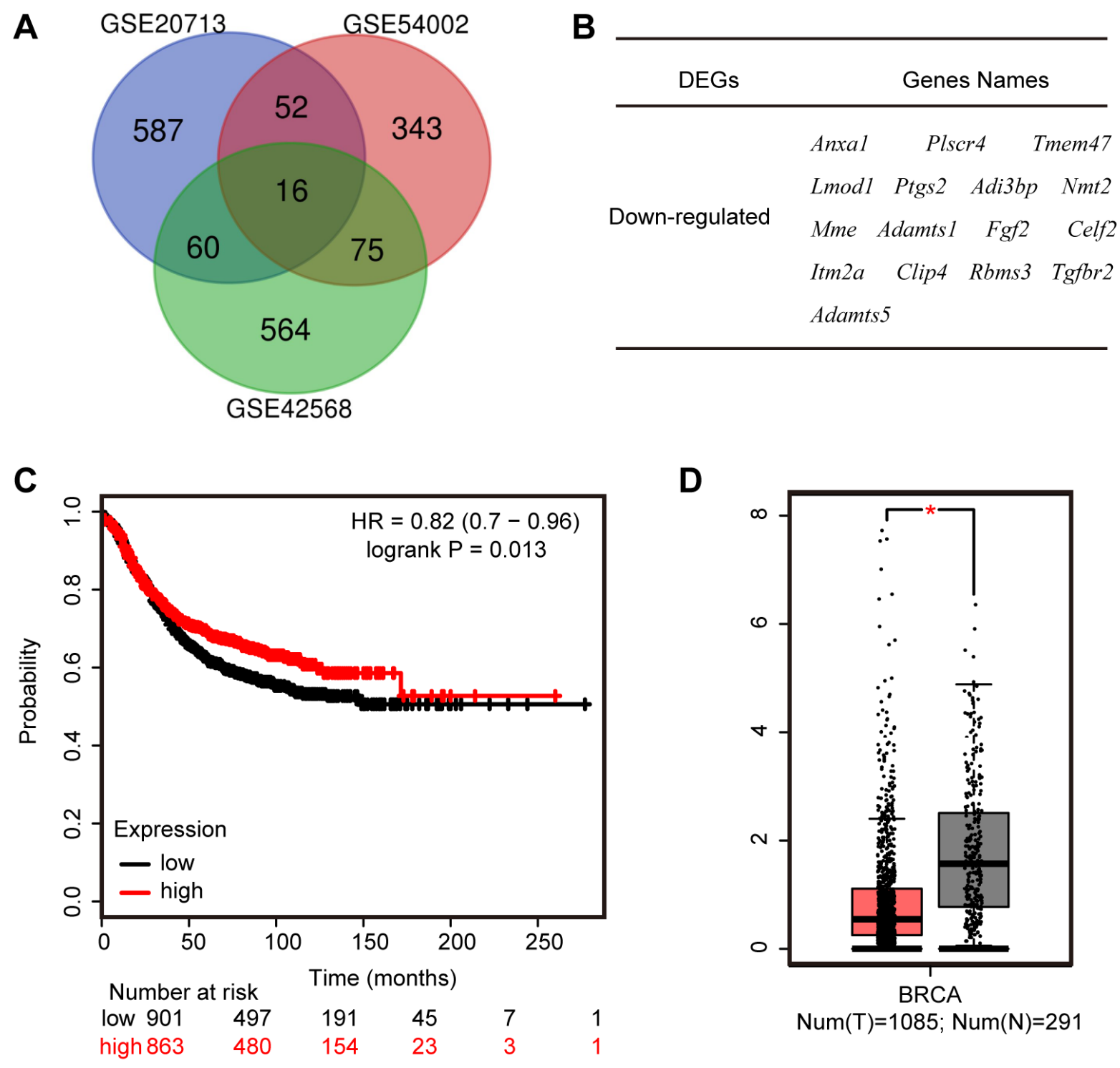

Figure 1 
Low Expression of Ptgs2 in Breast Cancer. (A and B) Authentication of 16 down-regulated genes $(\log \mathrm{FC}<$ -2) in breast tumor compared to normal breast tissues from the three datasets (GSE20713, GSE54002 and GSE42568) by Venn diagrams. (C) The prognostic information of Ptgs2 was showed by KaplanMeier plotter. (D) Gene Expression Profiling Interaction Analysis (GEPIA) for the expression of Ptgs2 in tumor tissues (red box) and normal tissues (gray box). T, tumor tissues; $\mathrm{N}$, normal tissues. ${ }^{*} \mathrm{p}<0.05 \mathrm{vs.}$ normal tissues.

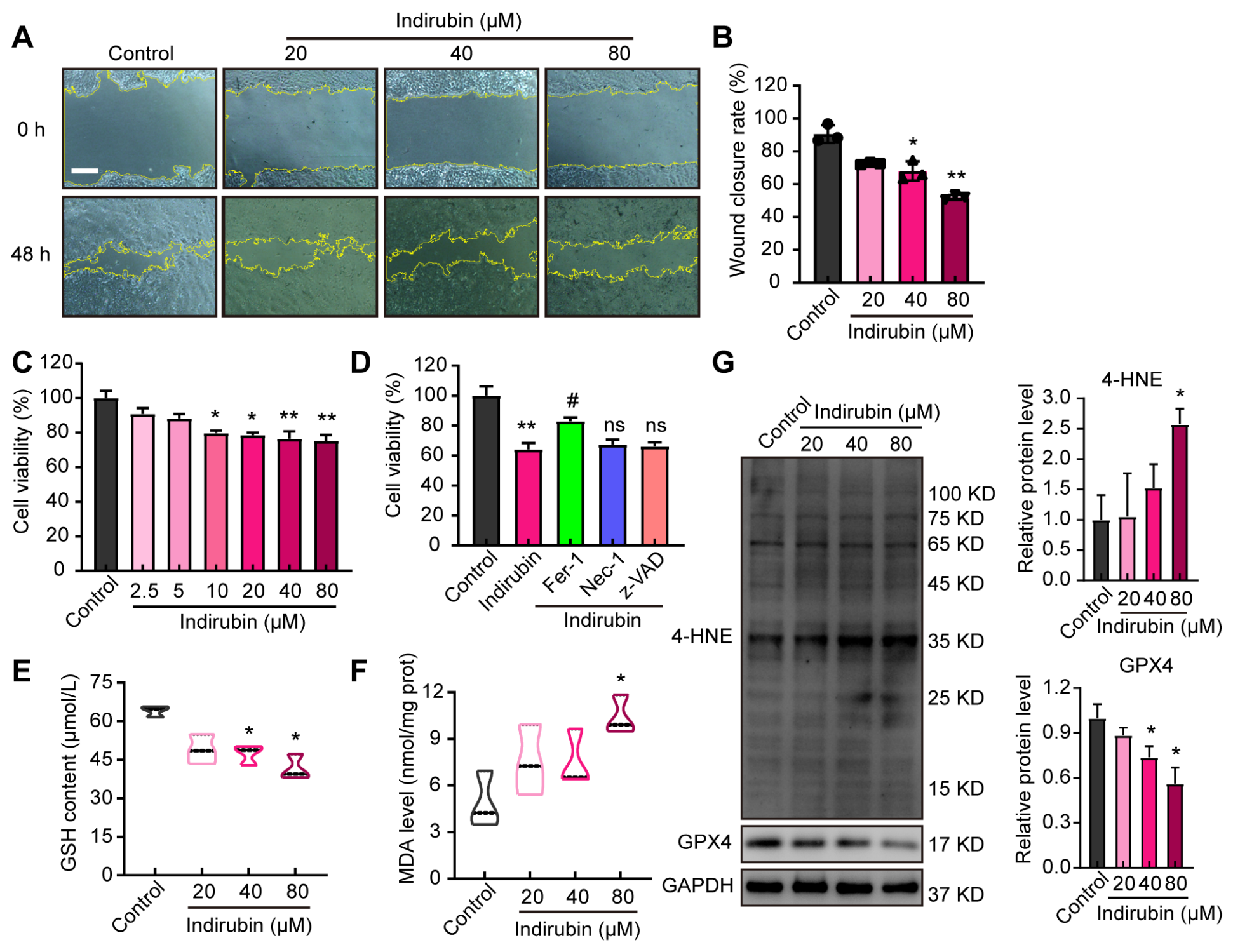

\section{Figure 2}

Indirubin induces ferroptosis of breast cancer cell in vitro. (A-B) Representative images of cell migration and quantitative analysis of wound closure rate. Scale bar: $200 \mu \mathrm{m}$. (C) The viability of 4T1 cells with indirubin at indicated concentrations for $24 \mathrm{~h}$. (D) The viability of $4 \mathrm{~T} 1$ cells pretreated with Fer- $1(25 \mu \mathrm{M})$, Nec-1 $(50 \mu \mathrm{M})$ or z-VAD $(50 \mu \mathrm{M})$ and then with indirubin $(80 \mu \mathrm{M})$. (E) The GSH content of $4 \mathrm{~T} 1 \mathrm{cells}$. $(\mathrm{F})$ The MDA level of 4T1 cells. (G) Western blotting detection for 4-HNE and GPX4 and quantitative analysis. ${ }^{*} p<0.05,{ }^{*} p<0.01$ and ${ }^{* \star *} p<0.001$ vs. Control group; ns (not significant), $\# p<0.05$ vs. Indirubin group. 

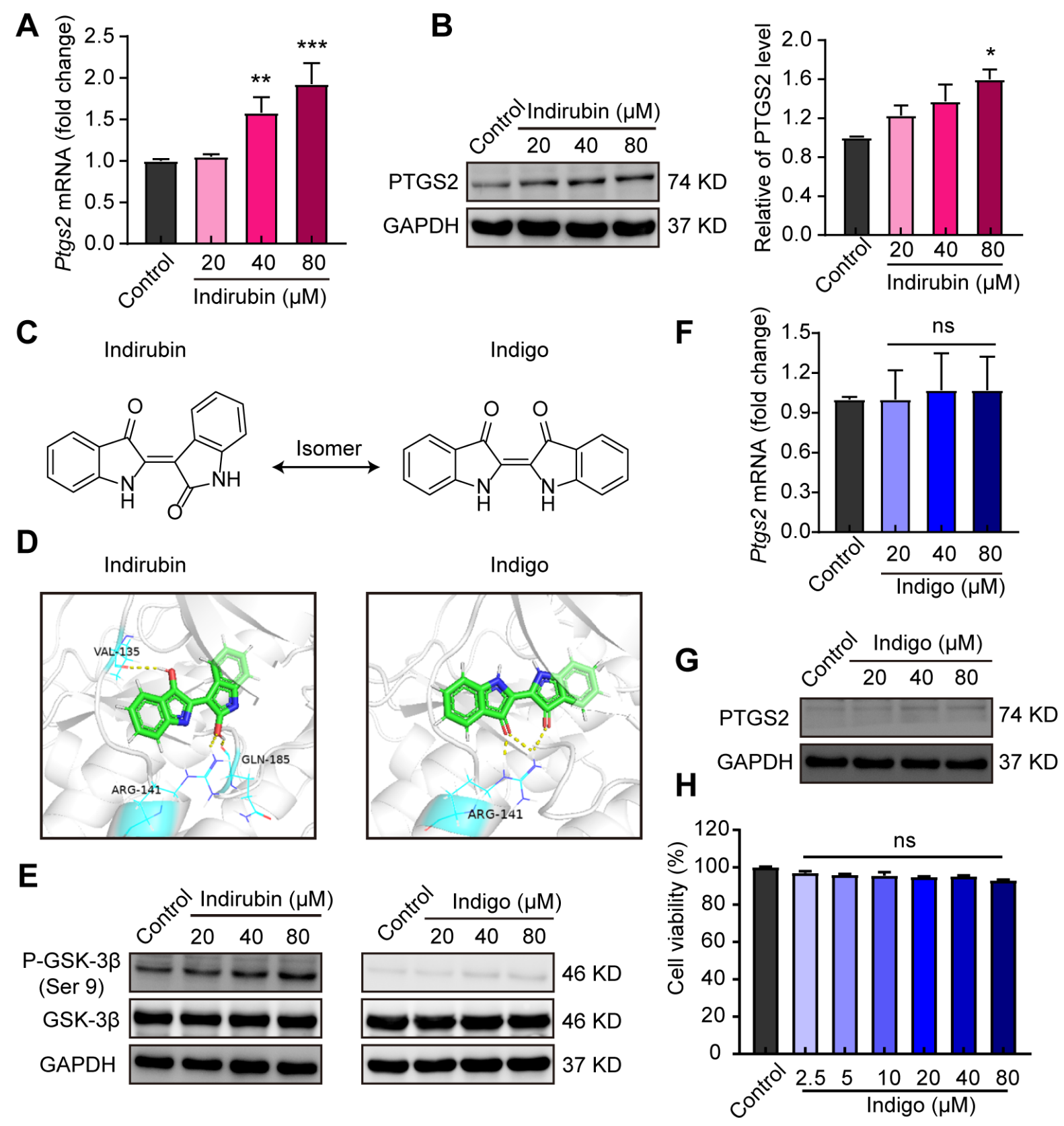

Figure 3

Indirubin up-regulates the expression of PTGS2 in breast cancer cell. (A) The mRNA level of Ptgs2 in 4T1 cells with indirubin. (B) Western blotting for PTGS2 in 4T1 cells with indirubin and quantitative analysis. (C) Chemical structure of indirubin and indigo. (D) Molecular docking of indirubin and indigo with GSK$3 \beta$. Yellow dot line indicated interaction of hydrogen bond. (E). Western blotting for P-GSK-3 $\beta$ (Ser 9 ) and GSK-3 $\beta$ in $4 T 1$ cells with indirubin or indigo. (F) The mRNA level of Ptgs2 in 4T1 cells with indigo. (G) Western blotting for PTGS2 in 4T1 cells with indigo. $(\mathrm{H})$ The viability of $4 \mathrm{~T} 1$ cells with indigo at indicated concentrations for $24 \mathrm{~h}$. ns (not significant), ${ }^{\star} p<0.05,{ }^{* \star} p<0.01$ and ${ }^{* *} \mathrm{p}<0.001$ vs. Control group. 

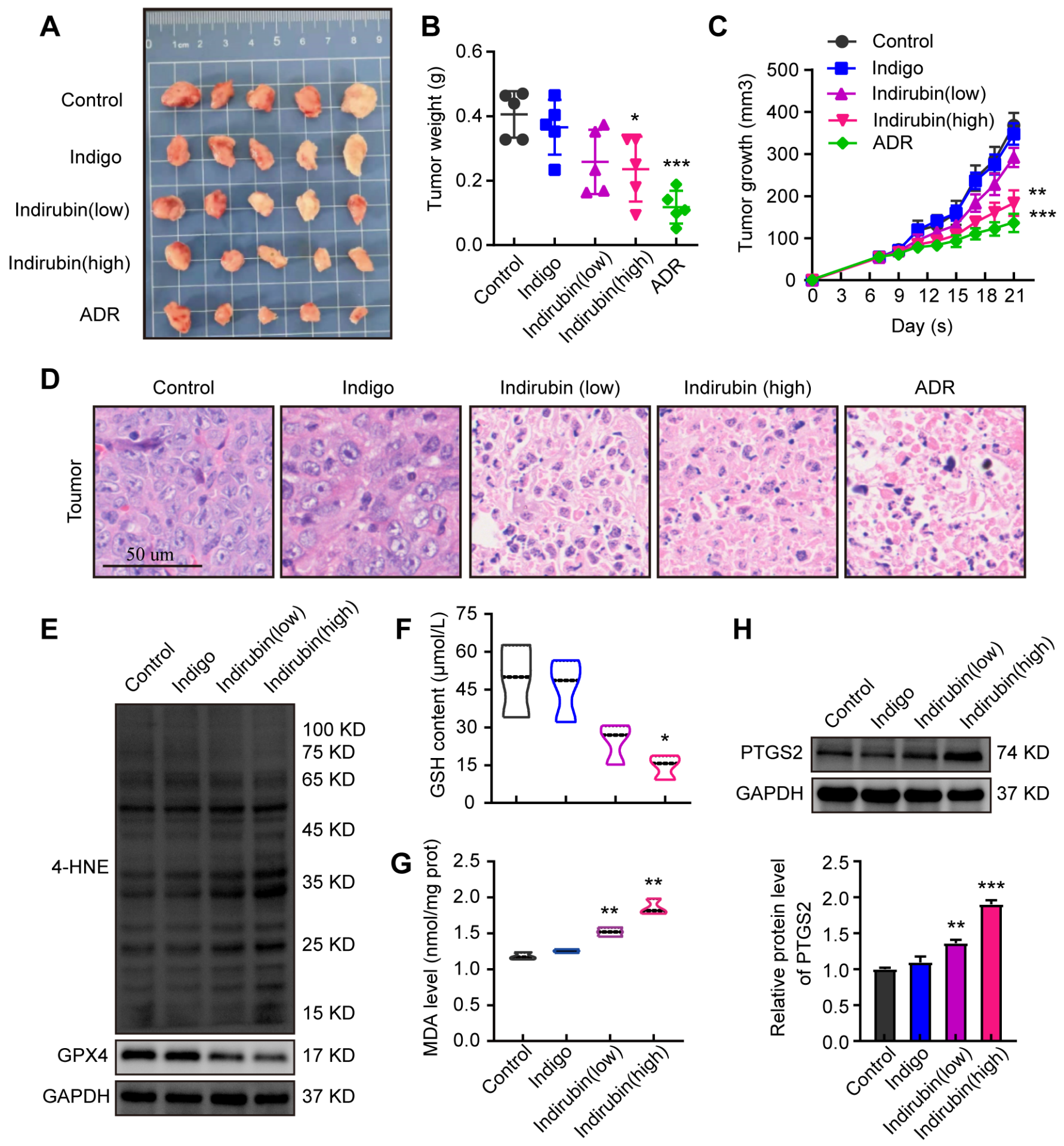

\section{Figure 4}

Indirubin suppresses breast cancer in vivo. (A) The picture of tumors in different groups of mice $(n=5)$. (B) Tumor volume of mice in each group was determined at the end of treatment $(n=5)$. (C) Tumor growth curves of different groups of mice ( $n=5)$. (D) Histological images of tumors sections stained by H\&E. Scale bar: $50 \mu \mathrm{m}$. (E) Western blotting detection for 4-HNE and GPX4. (F) The GSH content of tumor tissues. (G) The MDA level in tumor tissues. $(H)$ Western blotting for PTGS2 protein and quantitative analysis. ${ }^{*} p<0.05,{ }^{\star \star} p<0.01$ and ${ }^{\star \star \star} p<0.001$ vs. Control group. 


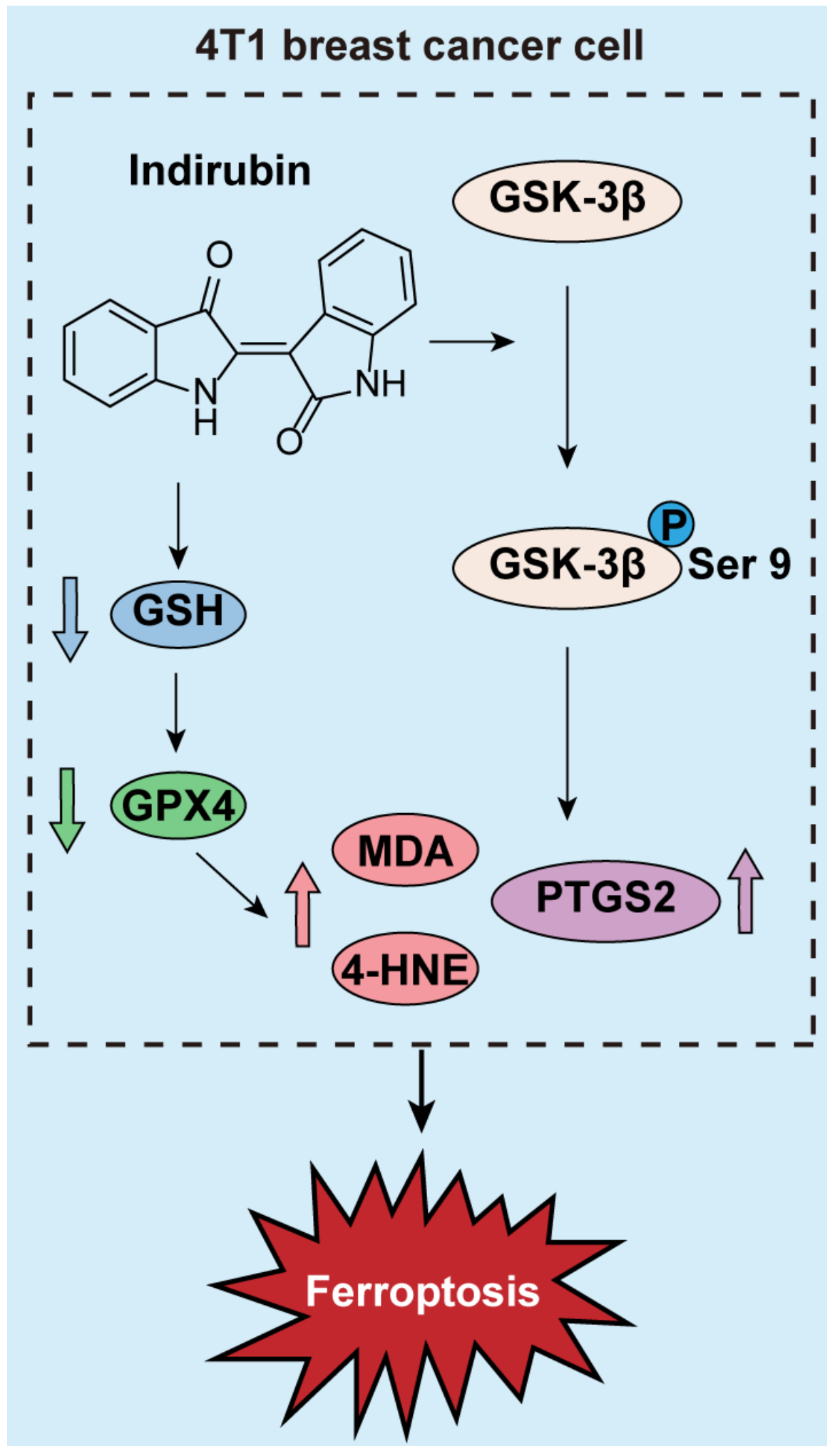

Figure 5

The mechanism diagram of indirubin against $4 \mathrm{~T} 1$ breast cancer model in vitro and in vivo.

\section{Supplementary Files}

This is a list of supplementary files associated with this preprint. Click to download. 
- Additionalfile1SupplementaryFigure101.tif

- Additionalfile2SupplementaryFigure201.tif

- Additionalfile3SupplementaryFigure301.tif

- Additionalfile4SupplementaryFigure401.tif

- Additionalfile5SupplementaryFigure501.tif

- Additionalfile6SupplementaryTable1.docx

- Additionalfile7TheARRIVEchecklist.docx 